\title{
Proceedings from the Document Academy
}

Volume 8

Issue 1 Robert Pagès: Documentary

Transformations and Cultural Context

Article 2

2021

Documentary Transformations and Cultural Context

Robert Pagès

callipie@yahoo.com

Follow this and additional works at: https://ideaexchange.uakron.edu/docam

Part of the Library and Information Science Commons

Please take a moment to share how this work helps you through this survey. Your feedback will be important as we plan further development of our repository.

\section{Recommended Citation}

Pagès, Robert (2021) "Documentary Transformations and Cultural Context," Proceedings from the Document Academy. Vol. 8 : Iss. 1 , Article 2.

DOI: https://doi.org/10.35492/docam/8/1/2

Available at: https://ideaexchange.uakron.edu/docam/vol8/iss1/2

This Article is brought to you for free and open access by University of Akron Press Managed at IdeaExchange@UAkron, the institutional repository of The University of Akron in Akron, Ohio, USA. It has been accepted for inclusion in Proceedings from the Document Academy by an authorized administrator of IdeaExchange@UAkron. For more information, please contact mjon@uakron.edu, uapress@uakron.edu. 


\section{Translator's Note}

The following is a translation of "Transformations documentaires et milieu culturel (Essai de documentologie)," a paper by Robert Pagès, published in Review of Documentation in 1948 (volume 15, issue 3). Translation by Michael K. Buckland with assistance from Olivier Le Deuff and Arthur Perret.

In this translation, Pagès' rather idiosyncratic orthography has been retained. His use of capital letters for emphasis has been rendered in italics, but his frequent use of boldface type has not been retained. His footnotes using Arabic numerals have been preserved. Explanatory endnotes using Roman numerals have been added, and a few references have been amplified. Square brackets indicate [editorial insertions].

\section{Introduction}

The point of departure for the following observations is the question of the relationships between the functions, domains and certain operations typical of documentalists and librarians.

\section{Documentalist and Librarian}

In the present state of accepted ideas, this is a problem of documentology, the study of documents. This formulation of the problem makes apparent the dissymmetry between what relates to the book and what concerns the document in general: the documentary domain includes or subsumes the librarian's domain.

\section{Cultural Technology}

On the other hand, this question leads, in its development, to the integration of nascent research on documentology within a system of research that is simultaneously vaster, older, and, so far, less systematic: that of the theory of human culture considered in its evolution. Documentation is to culture what machinery is to industry. And there is nothing more important for the study of culture than the analysis of its technological base, which is more and more equipped, regulated, and methodically organized. (Aesthetics, the modern economy, and the analysis of modern warfare show how far the technology of a specific social activity helps to make it intelligible.) Clearly, many methods, psychological, linguistic, sociological, logical, etc., should be concentrated around this study, as always when one wants to explain something in the humanities. 
Cultural technology itself can hardly pass as anthropologically insignificant except in the eyes of certain spiritualists who suppose that ideas, like babies, are brought by storks, or some "materialists" hypnotized by the industrial technique to the point of neglecting other techniques and really treating culture only as a "reflection" of it. Without explaining here a critique of those two methodological attitudes, I will only try to suggest by my conclusions the anthropological reach of an analysis of the structure and evolution of the documentary technique.

This evolution is marked notably by the higher position of the professional role of the documentalist relative to that of the librarian: one will have to investigate the meaning of that fact more deeply in the effective relationships between the book as an instrument and the document as an instrument, each with respect to these professions.

\section{The Opposition of Book and Experience}

Preparing definitions by examples for the purpose of induction, let us recall a classic antithesis which for centuries has enlivened the average opinion on culture: One opposes books and life. The "one" is, largely, the social sector not specialized in culture. Sentiment established an "association" (often enough valid) between the dust of books (a symbol of old age and decomposition) and the library user ("bookworm"). At the beginning of a cultural revolution, Descartes contrasts the dusty books with the "book of the world," which he preferred. He emphasized, not with his words but with his conduct, the antagonism between lived experience (direct and personal) and the teaching of books: It is not by chance that, to get away from the bookish heritage, Descartes was constrained to travel, wage war, join organizations, and so on. In the 17th century there was, as a teaching instrument, almost only the book; ${ }^{1}$ to break with book content, it was necessary to break with education itself, with the tradition of the linguistic form. But the techno-scientific character of modern culture has broken loose only through experimental practice, itself a break with the bookish document. This "empiricist" anti-bookish spirit has extended to ethics up to the present and in current literature (Goethe, Gide: "Theory is gray"; "Toss the book." )

Nevertheless, we will have to examine whether the antagonism between experience and book continues in the new and more general form of experience versus document or if, justifiably, the generalization of documentary form, with the proliferation of its particular forms, does not tend to cast off the symbolic dust from the document and to put it, not into opposition, but into a direct continuity with

1 There was also correspondence, sometimes organized [e.g., by Marin] Mersenne (1588-1648)], anticipating periodicals. 
experience. This suggests that documentary forms extend into a more or less unoccupied zone between fixed verbal language and lived experience.

\section{Accepted Definitions in Documentology}

\section{Book}

One need hardly define a book, it seems; nevertheless, to allow useful distinctions, let us say that a book is a fixed assemblage of leaves principally carrying written signs, that is to say symbols derived from language to be visually fixed, an assemblage reproduced in a large number of copies, usually by a typographic process.

An assemblage of loose leaves is not a "book," nor is an uncopied manuscript or an album (without written content)...

\section{Librarian}

The librarian is originally someone who does the job of all the operations concerning a book depository: collecting and conservation. This definition is intentionally incomplete. The function of the librarian in fact achieves its full development only through its response to the general documentary technique, which is newer than it. And, in fact, it has only been able to develop by itself giving birth to non-bookish kinds of document: moveable slips and cards (Abbé Rozier, France, 18th century ${ }^{i i}$ ) which, on the other hand, have become generalized and emancipated, and have assumed independent functions. The librarian is already, as such, a documentalist because he creates and manages non-book documents. To tell the truth, the real collecting and conservation are mostly done by workers who are not librarians...

Having used the immediately evocative powers of the word "document," now is the time to define it and its family of derivatives.

\section{Document}

A document is, according to UFOD [the French Union of Documentation Organizations] (whose point of view has spread internationally) "any materially fixed basis for knowledge capable of being used for consultation, study or proof."

The formulation confirms the fact that, since fairly recent times, all "bases" for knowledge other than the book, or more generally, print, are used for "consultation, study or proof" and systematically at that: namely, non-textual 
graphic media, pictures (iconography generally), automatic iconography fixed (photography) or moving (cinema), three-dimensional works (from stereo images to sculpture and immoveable documents: monuments). Let us add, and this is the main point, as one will see, following the questions sketched above, documents which do not result from a representation of an object but which are directly themselves the object able to inform about itself (living or dead animals, plants). One knows, however, that such a document is constituted as such only by the addition of an auxiliary document, of a sign, usually textual, which integrates it into a documentary ensemble, or, if you like, a contextualized document.

\section{Documentation}

Documentation is presented by UFOD as the set of operations concerning "the organizing, search, collecting, and utilization" of documents as defined above. It is necessary, therefore, to include, for example, bringing to light three-dimensional documents or objects (geological, paleontological, prehistoric, etc.) capable of constituting documents. There will be reason, on this point, to examine the relationship between documentation and archaeology, for example. Equally, the preparation and conservation of all material forms of document.

\section{Documentalist}

The definition of documentation clearly implies that of the documentalist, the professional agent for all documentary operations.

Comment: There is no necessity that documentary operations, already divided within the definition, all be performed by a single professional group. It is natural that this would be somewhat so at the beginning, in a phase of proliferation of tasks without specialization. But there is no reason for a division of labor resulting from an analysis of operations not to develop in this domain: documentalist designates virtually a professional ensemble or a genre, like "railroad worker." In the present state of the technique in general, it could be that the term documentalist tends to designate the function of operational integration, or coordination, or of directing. Now UFOD is training documentation assistants. T. Leroy reports the distinction between a classification maker (qualified classificationist) and a classifier (who applies a classification): each only performs segments of documentary operations. ${ }^{\text {iii }}$ 


\section{Documentary Technique and Representational Technique: A Critique of the Definitions}

\section{Document}

It seems that in the proposed definitions there are some ambiguities of little immediate practical consequence, but of some theoretical importance now and of practical importance soon. It is necessary to apply to them, as to every definition that affects the outcome of subsequent reasoning, the most critical analysis.

The most essential point seems to me to be the expression "basis for knowledge" used for "document"; (a rather practical consequence of this point concerns encyclopedic and specialized classifications). One can use the term knowledge [connaissance] in two senses:

1. Strict: Science, that is to say, generally, a mathematical expression, or mathematical-experimental, or mathematical-experimental-logical and, in a strict sense, logico-experimental (young sciences).

2. Broad: All that can be learned or taught, including the catechism or aesthetic, affective culture. Undifferentiated, global "knowing," like the Veda (same root as the Greek oida, knowing) or the Bible (a single book). In the first case, one ends up at a "scientific" definition of document, of documentation, and of the documentary profession: The documentary whole amounts to a scientific encyclopedia. This is not the case in fact, although encyclopedists, even today, very rarely express encyclopedic points of view that are explicitly non-scientific (artistic, technical, political...) and they nearly always mix many points of view under the heading of "knowledge" in the vague sense (the second sense).

In the second sense, which is clearly the relevant sense, the term knowledge is ambiguous (the first meaning is its "harmonic" sound) and does not usefully suggest the variety of cultural uses of documents.

But the term basis introduces through its vagueness a real error: any fact can be the basis for knowledge (in all senses of the word) and be both physically fixed and susceptible by its presence to be perceived - without thereby being a document.

\section{Document and Symbolization}

The vagueness of the term "basis," like that of "knowledge," conveys in fact indecision over what makes the shared characteristic of documents and over what one knows nevertheless exists because it is implicated in the analogy of operations on documents.

An additional concern is introduced by the limitation, also somewhat 
scientific, that is suggested by these operations: The Legend of the Ages (a book) ${ }^{\mathrm{iv}}$ or L'Estaque (a painting by Cézanne), which are documents, are susceptible to being used "for consultation, study, or proof," but they are principally used for something else (contemplation, aesthetic reading). Their ability to be possibly and secondarily used "for consultation, study, or proof" does not indicate that they are documents: one can consult a doctor, study a sick person, prove by witness: and what is not "materially fixed"? In fact, the suitability of the objects mentioned results from the fact that they already have the characteristics of documents.

Anyway, if it is possible to study anything, it is not possible to "consult" anything, nor to "prove" by anything. One consults (and proves by) two kinds of things:

- humans, for whatever they currently express (one can still sometimes include oracles)

- documents inasmuch as they materially fix (independently of humans) an expression or something suitable to being treated like an expression.

The UFOD definition is made by using skills as if competencies: It seems good because documentalists half-understand it. It is what it does that is bad.

\section{Symbolic Activity}

To define a document, it is necessary to introduce a psychological notion, that "of an expression or something apt to be treated as an expression."

Every object whose principal use is to be interpreted, that is to say, to serve as the psychic equivalent or reminder of other objects ${ }^{2}$ is a sign or a symbol ("symbol" is used in the contemporary sense, where it is not specified whether it is conventional or not).

Note that all perception is made from such interpretations: the smallest fork is treated as a "symbol" every time one shows that one "understands" it by the use one makes of it; but the use one makes of it is not, in essence, to "interpret" it: It is essentially a tool, not a symbol.

It becomes a symbol if, in an exhibition, it serves primarily to evoke the domestic equipment of the 18th century.

All the transitions can exist: A modern officer's sword, is it a weapon or a symbol? (And the horse, what is that?)

But for a whole set of objects, the non-interpretive actions that affect them (e.g., burning books for heating) are clearly less important than interpretative actions.

One can look for many explanations of "pure" symbolic activity. It will be

2 "Object" is used in the widest sense. It could be mimicry or a "state of mind." 
useful to state that, in most cases, symbolization (the production and use of symbols) replaces sensory operations or the manipulation of entire objects of the human world with various acts that are abbreviated, simplified, suggested... This implies that symbolization achieves thereby an enormous economy of energy, most especially muscular.

\section{The Duration and Accumulation of Symbols}

Certain types of symbolic objects endure because of their natural physical characteristics (stone monuments, medals). Others are unstable, such as oral signs (language) or mimicry, which do not outlast their expression. It is a fact that people have tried to accumulate their symbolic objects like other tools and products, in order to transmit them more or less further in time and space and thereby increase their effect. It is in this way that naturally stable symbolic objects, typically monuments (the dominant documents of the past), have been combined with naturally unstable symbolic objects extended by technology beyond their moment of expression (writings, drawings). It is of the latter that one readily thinks of when speaking of "documents."

The prolongation of duration can be artificial (intentional), but also natural and involuntary (or even "noluntary" [not-wanted]). It can affect durable objects that did not become symbols until after the event (fingerprints of a criminal or the imprint of a prehistoric cave-dweller's hand), or symbolic objects for primarily non-interpretative use (money, postage stamps, converted into pure documents by numismatics and philately).

But even in this case, deliberate conservation adds to accidental persistence and integrates the new documents into the documentary accumulation. Even if a document was not created as such, it integrates itself, even if very provisionally, into the documentary collection, as soon as it is treated as such.

A document is a symbol or grouping of symbols able to endure naturally or artificially beyond the moment of its expression (the degrees of this duration are very variable; from some minutes to thousands of years) and thus serve as an accumulation instrument for symbolic activity.

Whose symbolic activity? Nothing is said because it could be an individual or any group. (A “intimate" journal could be for an individual's use!)

\section{Symbolism and Documentology}

The analysis of the domain of documents is not separable from an analysis of symbolic activity as a whole because this domain envelops the first (documentary symbols + non-documentary symbols, without counting semi-symbols of all 
perception and action). This connection with documentology is not one-way, and symbolism should benefit from the results of documentology.

\section{Symbolic and Documentary Types}

\section{Elementary Symbolisms}

Documentary evolution clearly depends on the "material" which is initially provided by symbolization. It is necessary therefore to briefly characterize elementary symbolic material. There are two principal types: optical and acoustic (in relation to the preferred kind of human sensing).

The visual group appears particularly suitable, up to now, for providing almost complete substitutes for the perceived object (whether in three-dimensional or image form).

The acoustic group has developed music (essentially motor and affective symbols almost like direct stimuli) and language, bringing beside it a weak category of onomatopoeias considered "conventional" symbolism, which is to say not behaving in an automatic relationship outside a given social group.

\section{The Preponderance of Language and Writing}

The direct recording of acoustic symbols did not appear until long after the fixing of (often natural) visual symbols. Language was only fixed by its combination with the visual group which had achieved, from "ídeography" to the modern phonetic or semi-phonetic writing, the secondary symbolization of sound through visual symbols. This symbolization was quickly preferred and the written linguistic document has largely superseded the three-dimensional document; it has also made possible new cultural forms (science, literature). The predominance was so well established that in the 19th century history was defined by the use of written documents (Fustel de Coulanges ${ }^{v}$ ). Still in the 20th century the behaviorist John B. Watson could identify thought with "implicit" gesticulation of the vocal cords ${ }^{\mathrm{vi}}$ while other psychologists wondered seriously whether there was "thought" without language.

The domination of the written dates especially from the Renaissance, following the appearance of printing. But this invention itself had been possible only through the combinatory capacity characteristic of phonetic written signs that suggested moveable type. 


\section{The Evolution of Symbolisms}

It is here that one sees that the elementary symbolic material is not given once and for all. The purpose of writing is identical to the documentary purpose: to fix and accumulate symbols. But here documentation ended up creating new types of (audio-visual) symbols. The old types themselves, which had never been simple and pure, changed profoundly.

\section{Language and Its Double Floating Limit}

\section{Mimetic Contiguity}

Language, originally the preponderant branch of representation, never entirely separated from its border (boundary) with mimicry or with music, which conceals the imperfect written dissemination, which has contributed a lot to isolating language (the notation of modulation and stray sounds is very rare).

\section{Mathematical Contiguity}

But what is important is that language also engendered a "parasite": the domain of mathematical signs, arising from "punctuations" or from simple abbreviations and which presents some aberrant peculiarities. One can object that mathematical signs are perhaps not "symbols" like the signs of language, that they have another "essence"; what matters in practice is their genealogical continuity and indeed their current contiguity with language.

\section{Parallel Effects on Linguistics and on Documentology}

Linguistics can no more impose rules of good grammar than can language itself. Linguistics has common borders with the study of mimicry, with scientific "language" (including logic), graphology... It is a field impossible to separate precisely from the science of symbolism. One understands that documentology in its turn cannot exceed in rigor the symbolic material of documents. The fusion or differentiation of symbolic types leads to the fusion or differentiation of documentary types. The indeterminate boundary of linguistics correlates with that of linguistic documents and therefore also of librarianship.

\section{The Characteristics and Expansion of Mathematical Symbolism}

An essential exceptional characteristic of mathematical language is that the identity 
of the written sign does not necessarily entail the same sound in different languages. So one has here an international graphic "language" (more or less) in its particular signs and phonetic polyvalence (varying by nation). On the other hand, some sectors of mathematical language, if they do not sound the same, are susceptible to unambiguous geometric transpositions, which puts mathematic language directly next to iconographic and three-dimensional symbolism (diagrams). These characteristics are of an importance comparable to the extension of mathematical (or morphologically similar) procedures:

1. in anthropology and politics (statistics)

2. in sectors that do not admit measurement:

a) the substitution of figures for verbal concepts in organizational charts, Gantt charts...

b) the logical generalization of algebraic symbolism

c) the accelerated development of classification, using ordinal numbers directly or indirectly

d) establishing veritable languages (terminology) on a classificatory basis (e.g., the Universal Decimal Classification and the phonetic notation of G. Cordonnier, ${ }^{\text {vii }}$ which has predecessors from the 17 th century)

If one examines the meaning of particularities of mathematical symbolism, which absorbs natural language, one finds that it is more textual than phonetic. It seeks to use the combinatorial capabilities of written signs (a capability already particularly responsible for typography) to establish very abbreviated symbolic equivalents, hence very manipulable yet rigorous, of partial but precise descriptions of objects: semantic condensing. Historically the combinatorial output of mathematics goes together with the creation of abbreviated symbols (geometry among the ancients, Hindu-Arab positional numbering, the invention of zero, algebraic signs). Logic itself has advanced only by becoming formally algebraic.

One can then show in contemporary symbolism the growing development of combined stenographic and combinatorial skills, whose type of operation would have been the algebraization of geometry.

\section{The Fixing of Phonetic Language (Phonography)}

But at the same time, a seemingly opposing development is emerging: although the mathematical trend pushes towards maximizing certain basic characteristics of written language, this is losing ground before the direct fixing of language: phonography, films... Without insisting on this topic, one can see in it a reaction against the inexactness of the written reproduction of language and its replacement by the reproduction of language itself. 


\section{The Revival of Visual Documentation}

The reproduction of language itself is, even within the field of symbolism, only a symptom of a more significant tendency: that which pushes for the most exact and complete reproduction possible of the objects to be "represented" (symbolized). Hence the revival of visual documentation in all its forms, with a proliferation of new forms.

The texts of publications are completed with or replaced by images. An image which is synoptic and direct is generally more intelligible than a verbal explanation: It appeals to a more accessible level of intelligence; hence it needs less intelligence (an application of a known psychological principle).

I'm not insisting on diagrammatic images, which resemble mathematical forms. Note, however, that, as an instrument of abstraction, the diagram (whatever the degree of abstraction), retains a resemblance to the object. The advantage that the graphic equivalent of a concept has is that of exactness. The renaissance of the diagram is a revival of "ideography" beyond phonetic writing.

Photography is, like a diagram, characteristic of these tendencies: It has, on the whole, replaced the hand-made drawing for description, which is inexact, rare, and costly.

Just as typography, based on the reducibility of language to a small number of phonemes, made possible a predominately linguistic-written culture, so too the new expansion of ideography and iconography is and will be mediated by the techniques of the inexpensive mass reproduction of images (heliogravure and derivatives of lithography, offset, for example).

The massive reproduction of images is, historically, as effective a factor in change as printing was in its time.

The most original and independent form of iconography constitutes in fact a symbolism of a new type, although derived from photography: the cinema. The cinema is a domain of expression so developed that already presents two dimensions (at least!), an imitative dimension and a mathematical dimension: it includes, in effect, both the mathematically or mechanically animated diagram and the most detailed photographic image. 


\section{Quasi-Empirical Symbolization}

40 The above is a short review necessary for forming some hypotheses.

\section{Comment on the Role of Technical Inventions}

One of the basic laws derived from the history of technology is that an invention does not by itself determine its adoption. That depends on the local civilization and its receptiveness. ${ }^{3}$ The massive expansion of the techniques mentioned above reveals the presence of latent or overt needs which they have met. The relationships between invention, social adoption, and needs cannot be reduced to any form of causality, but consist of all kinds of relationships and interdependences which need to be determined one by one.

It would be as easy as it would be interesting to show the need for iconography or even for cinematography, before the cinema expressed itself, in the art or literature of the 19th century (romantic drama, realistic novels, the poetry of "visionaries"...).

Here I will take up the question from another angle, considering technologies as symptoms of needs and of tendencies that their adoption satisfies.

\section{Aspects of the Historical Role of Photography}

Let us specify some aspects of the role of photography which for a long time constituted a veritable social symbolic theme: that of the exactness in the visual reproduction of reality. Like fingerprints, it serves to identify individuals without ambiguity (the function of a portrait).

There has always existed an activity of iconographic description of reality, in particular painting, which clearly had that function until around the appearance of photography. Hence informative detail in pre-photographic painting is absent from post-photographic painting. The role of affective, subjective expression, combined with information in old painting, separates in modern painting, while nevertheless emphasizing its role of chromatic description (Impressionists' landscapes) in contrast with the black and white of photography.

(What would happen to hand-colored iconography in the face of a correct mechanical competitor? A support activity, one can conjecture.)

3 Cf. P. M. Schuhl. Machinisme et philosophie. Paris: Alcan, 1938; 2nd rev. ed. Paris: PUF, 1947. 


\section{General Role of Symbolisms as "Reproducers" of Experience}

It is here that the definition of the symbol, if it is good, should serve for something. If the documentary accumulation of symbols has the aim of perpetuating their use, there are cases where the symbolization of the object, having the purpose of compensating for its instability, can serve to conserve the most perfect possible equivalent, that is to say, reproduce it.

The "opposing" of "book" and experience does not signify the "opposition" of every symbol, hence of every document, to direct experience. There are some cases where one needs to unburden and remove one's symbolization of an object, and others where any removal constitutes a loss (exactly as in the reproduction of... a document). Hence symbols that are more or less abstract or concrete (complete, unaltered).

These simple facts have led to much ambiguity:

There is an idealist way to defend modern "subjective" art by saying that it is not to imitate nature or reality since "that exists already." ${ }^{4}$ But no real experience exists for all time, in every place, and for every individual. Its unreliability or absence in these three aspects creates a need for exact and enduring substitutes. Hence the value which the simple copy of reality has always had.

\section{Reproductive Symbolism and Branches of Culture}

\section{The Aesthetic Problem and the Classic Problem of Logic 5}

Such are the themes jointly evoked by documentology: What established relationship is seen (or "necessary") between "reality" and its diverse "expressions" (symbols)?

\section{Scientific Representation and Lived Experience}

In the context of the discussions of the Vienna Circle, Philip Frank and Moritz Schlick ${ }^{\text {viii }}$ notably indicated that science is not a copy of "reality" nor truth the correspondence of thought and object. It is only a system of symbols in unambiguous relationship with objects; that is to say, for example, that one can predict the effect of certain operations on a group of objects from the corresponding

4 This aesthetic would have its correlative if not its origin in a "genetic" psychology of perception as "interpretation." Gestalt theory has removed the scientific foundations of this position by showing that perception is spontaneously "organized."

5 These terms are taken to mean the of intentionally scientific study of art and of science. 
effect of corresponding operations on a group of corresponding symbols. But the same school has shown that concepts (and operations on them: problems, solutions) can only have a "sense" that they are capable of corresponding to "actions," to the resulting behavior accompanied by as complete as possible sensory effects. Thus, symbolic activity has meaning only through controlling behavior.

From this control, written accounts are established, formed from "protocol statements." But the "verification" which could eventually operate on these written accounts is in its turn only an operation in the symbolic domain. The written accounts can result from an inadequate symbolization; they can be altered, badly interpreted (all kinds of incompatible "experiences" have had written accounts and "testimonies"). It may be necessary to correct them.

So it is not inside the documentary domain ("coherence") that the factor which stimulates, verifies and gives meaning to science can be found, but only in the immediate lived experience (Erlebnis), neither symbolized nor, in the end, recorded $^{\text {ix }}$ (e.g., two black lines in the orange field of a lens).

Thus ascertainment is personal. All my confidence in symbolic tools and operations, insofar as it is not metaphysical or religious, is based on their powerful ability to integrate my current individual findings.

The symbolic-documentary domain acquires its meaning and scientific efficacy only through the unambiguous correspondence of symbolic behavior with non-symbolic behavior, connected to total individual experience. (I associate with behaviors all their objective relationships.)

One should not hide from oneself that the epistemology of the Vienna School is still, methodologically, more philosophical than scientific. I think, however, there is reason to use its conclusions as working hypotheses, for lack of better and while awaiting better.

Consequently, in the case of science, it is easy to explain that symbolic reproduction and the document that constitutes it would be an indispensable approximation of lived experience, in the temporary or complete absence of that experience.

It is even necessary to admit that the domain of scientific experiments is itself, with regard to experience outside of science, only a global symbol, a "rough model" of what is properly called experience, and much more economically handled. Finally, "trust in science" is obtained only thanks to the massive permanent testing of rational technological civilization. It is in this context that the individual "assertion" has its effect. 


\section{Symbolism and Experience in Non-Scientific Cultural Domains}

\section{Preliminary Comment on the Notion of Experience}

One has seen that the word experience has various meanings, and one should not be ambiguous about a word. The "lived" declaration according to Schlick is not very different from a signal (in the example cited: two black lines on a yellow background), that is to say a symbol. Generally, scientific "experiments," for all that is different from "knowledge" (a system of symbols), concern signs or equipment, are very simple perceptions (especially visual or auditory), quite analogous to a simple symbol.

But they do not have the psychological or technical role of a symbol. They are, through the construction of "experience," an endpoint, a "product" that spills out of the domain of symbols and which integrates itself into the frame of perception and ordinary behavior. ("I have seen Mercury as I have seen you.") By "position," the "statement" foreseen and prepared through an apparatus of symbols and interpretive instruments, appears as what was been sought through symbols.

Scientific experiment is characterized by the selection of privileged perceptions that are simple, unambiguous and few in number. One sees that it is only a sector differentiated by sensed and perceived relationships of the organism and the environment. The differentiation has, however, a history: The "statements" of taste and smell were heavily used in chemistry not long ago. Now taste and smell (themselves little separated from each other) form a transition from perception to affect. On the other hand, all perception (visual, auditory) generally includes associated affective influences. Science is proceeding to eliminate affect, this former mode of exploring the environment, from its domain. The activities that one groups under the name of "art" develop at will perceptions emphasizing their affective effects.

Modern art was dominated by written symbolism. Does it produce the same progression of symbolism "reproducing experience," especially visual, as in the scientific domain?

The facts appear to prove it. The expansion of the cinema is still much greater in the "artistic" sector than in the scientific sector. It is not surprising that, except in the case where symbolism constitutes a relatively direct and independent affective stimulus (music), the indirect or abstract symbol (language) would not have the same effectiveness and is recharged only through the frequent renewing of its connection with the lived experience for which it is a substitute. The effect of a symbol depends on the degree of its ability to substitute its content in art as in science (the affective effect being associated with the sensory effect, unless there is a successful effort to eliminate it). 


\section{Limits of the Effectiveness of Written Documentation}

Documentation that is primarily written can only barely serve a culture that is limited to an objectively narrow experience, which has little variety and is conceivable or even achievable only by the "internal" means of an individual or of a group weakly equipped for external exploration. Many of the traits of medieval culture answer to this characterization (inward character of a mystical verbal culture).

It is true that the printed written document later enormously expanded the material domain of the culture.

Cicero saw perhaps five or six philosophers, whose scholarship was very limited, and in a library one can see thousands. There are more things to learn in a library, now that printing provides such an abundance of books, than Cicero could learn in all his travels. (Perrault, 1688) ${ }^{\mathrm{xi}}$

This is what Perrault said to support the cultural superiority of the Moderns over the Ancients. But to the extent that libraries are only stores of ordinary language, they cannot exceed the limits of this language, which is only capable of simple, crude, and very approximate empirical description and simple and somewhat confused logical combinations (especially when they try to become complicated). The development of the stored mass and the use of language only ends in linguistic inflation and depreciation characteristic of dying scholasticism (against which Descartes, among others, was justifiably opposed).

Since that period two types of reaction to the inadequacy of language appeared:

1. The development of the experimental "demonstration" (Galileo, Bacon, Pascal...).

2. The expansion of a symbolism whose combinatorial output made it constitute a new logic and even initiated the construction of a new type of language: algebraic analysis, from Descartes to Leibniz, with their projects for universal language of an algebraic type, antecedents of modern logics and more. 


\section{The Massive Diffusion of "Quasi-Empirical” Documents}

\section{Social Factors}

Here the social factor of industrial and political needs intervenes, noticeable from the end of the 18th century, expanding in the 19th and 20th centuries and which need to resolve technically the problem of diffusing a culture of efficiency in the constantly multiplying masses, that is to say making the experimental element compatible with symbolic elements. The massive multiplication of direct experiences is most often impossible. It is then (especially in the 19th century) that representational techniques emerged which one could call quasi-empirical, supplying mass reconstitutions and reproductions ... historical, geographical, technological.

At a time of mass production (factories), mass politics (parties) and mass warfare (conscription and total war) there also arose in culture the documentary industry of surrogates for experience. Modern societies no longer try base themselves on competition ("capitalism") but on collective cohesion, which requires a general understanding of the technical and social context.

\section{From Photography to the More Complete Reproductive Representations}

Photography, as has been seen, marks most characteristically the priming of this trend to mass illustration.

In its very process, it reproduces a veritable psychological attitude, being the automatic recording of the luminous image, ${ }^{6}$ without direct subjective alteration; also, it was the emblem of "realism," that is to say the quasi-experimental tendency of documentation.

Photography however only recorded light values, but the same process that constitutes it leads to the reproduction of movement and then of colors (cinema, photography and films in color). Combining film with the recording of sound, the achievement now of three-dimensional movies (in theaters in Russia), the creation of television which, in parallel with adding a soundtrack to film, combines audio broadcasting with largescale video broadcasting, underlining an orientation of a whole group of representations toward the quasi-experimental document, toward mass production of almost complete original perceptual experiences.

6 Similar to devices for automatic recording of movements as graphs (traces), from myography [measurement of muscle contraction] to seismography. The reproduction of sound by recording it, as opposed to written notation, belongs to the same family of technology. 


\section{Quasi-Empirical Documentation in Education}

One can only mention the educational use of film (either animated or not, still projection or not) as a relative retreat of textual and verbal documentation. Already the new school levels for eleven- and twelve-year-olds in France show the progress of the iconographic sector in education.

"These programs imply that the idea of knowing how to read, write, and count will not be enough in a time when photography, cinema, advertising, illustrated books and newspapers are releasing a torrent of images ..."7

The cinema seems particularly suited to accelerate education: it is so used for rapid military and professional training.

An interesting trace of semantic genealogy, "document" is connected linguistically to Greco-Latin roots, doc, dask, dak: (docere, didaskô, didactique) and recalls an epoch when the accumulation and transmission of symbols had the role of assuring the cultural continuity of generations. Still today, pedagogy is an important user and producer in the documentary domain. It is even at the level of the child that psychology and so pedagogy have the most modern and most rational development. And it is perhaps here that the trend in the evolution of relationships between symbolism and experience is best indicated. According to the founders of "active school" and "functional education," teaching, that is to say the transmission of symbols, or of documents, is only effective when associated with direct personal experiences; attention to these are guaranteed only by the spontaneous interest of the child, according to his or her current needs. Education prepares for life only if there already is "a life" (Edouard Claparède ${ }^{\mathrm{xii}}$ ). So we participate in the destruction "from below" of a pure culture, without an empirical foundation, which creates, according to the degree of culture, a social division between the literate and others who are "a-documentary" and hence "uncultured."

The introduction of cinema into school is only a symptom of this cultural type, tied to that of the documentary type.

The connection of the new symbolic-cultural domain to experience is equally demonstrated in the massive and rather recent development of a veritable frontier zone of documentation.

\section{Specimens and Auto-Documents}

460 A "unique object" or a member of a collection of similars perhaps kept and used as a "document" about the domain to which it belongs, or even about its own objective properties as 1) an individual or 2) of a type.

7 L. Machard. "L'éducation visuelle et le filme d'enseignement." Films et documents No 3; 1946. 
In the second case it is matter of a specimen: an anonymous Egyptian mummy, a gorilla in a cage, a piece of rock crystal.

In the first case the document transmits information about itself (Napoleon's hat, a unique meteorite). It is, therefore, an "auto-document." In fact, it is so only through association with verbal or more elaborate symbols (name, dates, historical description, classification code) which connect it to different symbolic sets.

\section{Museums}

These two types of objects are the principal constituents of museums whose development since the end of the 18th century goes in step with that of quasiexperimental documents.

But there is a continuous transition, here again, between museum and site of direct experimentation: Such is the Palais de la Découverte [Palace of discovery] in Paris, a permanent exhibition of experiments and specimens, where the ambiguity or the stages of science have been seen.

\section{Exhibitions}

Likewise most exhibitions are on a continuum between commercial use of objects (a fair) and their documentary use.

\section{Involuntary Documents}

Awareness of this ("fair-exhibition") transition only systematizes a spontaneous process of psychological documentary investment applied to non-symbolic objects: a diffuse base of the average cultural superiority of city-dwellers over rural, of equal education, is in this natural function of fair-exhibition and living museum which fills a city.

\section{Diffuse Documentation and Tourism}

The expansion of documentation, especially by the state, pushes the systematization this type of process much further: It "documentifies" (transforms into museums and living spectacles) places or habitations which it classifies, plant and animal species, and sometimes human culture, behavior, or races whose conservation it favors. In this last case, ethnographic documentation resembles zoological documentation and makes an enduring and passive document of mankind itself... Tourism, long a normal part of the education of the upper classes, is expanding among the masses (paid vacation, youth hostels) and tends to associate 
every human habitat with a secondary function as a documentary library. ${ }^{\text {xii }}$ Like an animal in a zoo, the hillside village of Rocamadour or a shipyard has the documentary label of a mention in a guidebook or publicity in a local newspaper (announcing the launching of a ship).

\section{Related Intellectual Changes}

The development of this semi-documentary transition zone is further proof of the reduction of the traditional opposing of the "life and experience" domain and the "culture and document" domain. Like modern education, culture tends to be "a life," that is to say a multiplication for the individual of vicarious experiences presented by the new documentary techniques. One cannot deal here with the reinforcement of this tendency by non-cultural activities. Simply, the formal texts of the intellectuals as an instrument of a metaphysics without empirical content and emptied of affective-mythic content, seem to be on the way out.

One can pause only for a moment to deplore that intelligence "is lost" as image and sensation gain. Lively and authentic images and sensations provide only resources for a type of intelligence that is different from the intelligence of [textual] phrases, a type that is certainly less conjectural, more rapid, more extensive, and more connected to practice. Too bad for intelligences of the ancient model which makes their loss of status a matter of "barbarism" and "civilization." But the most serious social-cultural issue is not there.

\section{Correlative Progression of Quasi-Empirical and Combinatorial Symbolisms}

\section{Combinatorial Symbolism}

I have, however, indicated that the quasi-experimental symbolism extends only at the same time as a mathematical type of representation of a seemingly "pure symbolic," extra-empirical tendency whose principal function is in relationships.

That signifies that the new culture continues to develop the specific properties of symbolism and not merely to reproduce and disseminate a growing mass of received experiences.

This trait is found again within the "quasi-experimental" documentary techniques. Photography and cinema are instruments of observation which produce new experiences because of their ability to vary the points of view of observation and its modalities: monochromatic images, angles of view, slow motion, acceleration, enlargement...

One can distinguish here: 1) the role of a sensing instrument (e.g., 
microscope, telescope), and 2) the already remarkable synthesizing role: direct experience (the relationship of an event and a witness) is a single point in space and time, perceived unilaterally or by slow successive exploration from different angles; the cinema already achieves selections (extracts...) and combinations (varied order, summary presentations) that require mobilizing a single image (montage). One recognizes the familiar operations of language, but achieved with images that are generally "mimetic" and not with words.

This type of properties goes together, for symbolism, with the degree of abstraction performed on experience: algebra retains nothing of something if it is not, for example, that "something" is "variable," "nonzero"... or whatever. Abstraction signifies selection and elimination; this type of properties also goes together with the degree of reduction and standardization tendancies of symbolism itself.

It exists only to a very small degree in language (as historical experience has shown even before logical analysis).

\section{Correlation of Combinatorial Symbolism with Empiricism}

Symbolism did not develop unilaterally towards experience, but it seems that between specifically combinatorial symbolism and experience a relationship develops that is analogous to what exists in a factory between the quantity of raw materials to be processed and the necessary machinery. That is to say that not only the quantity of abstract documents increases, but also their combinatorial product must increase.

\section{Reduction of Language}

This is how combinatorial symbolism ${ }^{8}$ is always moving further from the original language, which, on the other hand, has seen its descriptive function diminish. Although there are other factors which affect its fate (ordinary language remains a shared auxiliary language between specialists in different fields), ordinary language tends to persist only in less developed cultural areas, always subject to the pulling and fragmenting at its descriptive and logical extremes.

\section{Differentiation of Cultural Levels}

Now, combinatorial symbolism, unlike quasi-experimental documentation, is very

8 It is necessary to understand by that mathematics and logic, but also classification and mechanized selection ... 
weakly diffused among the masses. This diffusion is opposed by its growing diversification into "cultural languages," which are more and more numerous, all archaic and sterilely complicated, and for which the apprenticeship is interminable.

The literate-illiterate dichotomy, which dominated former cultures, is replaced by an opposing of the empirical-verbal level and the empiricalcombinatory level. In this way, what was, at least provisionally, the utopia and mystification of universal instruction is dissolved. The "masses" become well educated, but not into the (principally literary) culture that was assumed, and not even the same culture as the ruling (or ascendant) class of the industrial and social technocracy. One of the principal traits of the science that dominates contemporary culture is the inverse of the prescientific 17th and 18th centuries, the inequality among people in terms of experience, which has been translated into a specialized institutional hierarchy. ${ }^{9}$

This is important for anyone who doubts that cultural technology is in the process of becoming the socially decisive technology in contemporary society.

\section{Situation and Divisions of Documentary Techniques}

60 If one now returns to the particular problem that has served as our point of departure, it could be resolved secondarily through the notions established above. The present role of documentation, of the documentalist, and of their variations cannot be apparent without analysis of the internal structure of documentation as a segment of culture.

\section{The Function of the Documentalist}

One recalls the definition of documentation as the set of operations concerning "the identification, search, selection, ${ }^{10}$ and utilization of documents," which was intended to designate the professional operations of documentalists.

This definition calls for some reservations:

\footnotetext{
9 See, on this point, Gaston Bachelard, La formation de l'esprit scientifique. Contribution à une psychanalyse de la connaissance objective, Paris, Vrin, 1938. Transl.: The Formation of the Scientific Mind: A Contribution to a Psychoanalysis of Objective Knowledge. Manchester: Clinamen, 2002.

10 Concerning the definition criticized, note the ambiguous formulation: Not every case of the selection of documents has a documentary purpose; it could be an auto-da-fé. [Perhaps a reference to book burnings.]
} 


\section{It Is Not Documentary Production or Information}

One can "establish" documents (write a poem or a theorem) without thereby acting as a documentalist. The documentalist establishes documents which normally do not add to the intrinsic content of the documents on which he works.

Because, and this is an ambiguity that is as difficult as it is important to elucidate, if one wants to remain both coherent and credible, documentation operates on documents and not on direct experience.

Whatever continuity is established between documentation and lived experience or observation of the world, documentation in the sense of the work of the documentalist is not involved in the activities which establish the direct practical or theoretical relationships with experience. A video or literary reporter is not a documentalist. He requires some information, that is to say that he establishes documents on the news. Likewise, the development of documents for defined purposes is not the domain of the documentalist if the purposes are other (scientific, artistic) than those defined below.

\section{The Documentalist as Cultural Distributor}

In fact it is the general cultural use of documents that drives documentalist operations.

The documentalist appeared quite recently with the development of documentation centers, especially in the years immediately following the war of 1914-18, at the same time that a vast cultural concentration was taking place almost everywhere, corresponding to the monopolistic or state financial concentration accentuated by the war.

Scattered elements of the documentalist preceded the documentary reconstitution. The "pre-documentalist" occupations (librarian, archivist, administrator of museums and monuments, of zoos, ...) existed in semi-artisanal forms. A property of modern documentation is to coordinate its sectors in the same organization, as the modern economy coordinates several segments of the preparation and consumption of a product.

\section{From Librarian to Documentalist}

On the other hand, one has seen that the very structure of documents has changed. The dominance of the book was such until the 20th century that the librarian was the principal distributor of documentation. ${ }^{11}$

11 Note that there has always been a commercial sector of distribution (the book trade) which 
Already, however, its distributing activities have had to adjust to specialized production: the elaboration of bibliographies, of catalogs, and, soon, of card files. With the resurgence of, especially, visual forms of documents, the principal documentary store (the library), the principal documentary product (bibliography), the principal documentary profession (librarian) are no longer sufficient to assure distribution. The mass of books itself requires new procedures on the part of the librarian. Renan already considered that libraries would be unusable in a few years.

On the one side, document centers arise with an unlimited series of different resources (phonographic disc collections, etc.). On the other not only bibliographies, but documentographies are being developed with an open-ended series of different media (filmographies, etc.).

\section{Auxiliary "Documentary Productions"}

Finally, the documentary distributor responds to the inability of users in the face of the mass of documents by developing operations of simple storage and transmission with innovative enhancements added to the primary documents, which creates series of auxiliary documents to support use. These "documentary products" go from descriptive documentographies, simple lists of the civil status of documents, with analysis, selections, extracts, and even, if wanted, to reports, monographs, treatises and encyclopedias. These are documentalist-type operations in that they do not produce and do not aim to produce new documentary "content." In principle, the documentalist does not act on lived experience and does not produce original documentary content. Whatever the mixed forms and their eventual development might be, it is important to define the state of the current situation. Not yet mentioned is that not every non-original producer is thereby a documentalist: notably, he could be an educator if producing textbooks: in this case, distribution is associated with a specialized technique.

\section{The Combinatorial Operations of Documentalists}

That said, it is equally important to indicate how much, for example, documentographic selections using punch cards ${ }^{\mathrm{xiv}}$ can encroach on the hitherto unautomated processes of productive document use (bringing information to inventive effect). The distribution of all genres is not always as "unproductive" as metaphysics and morality would sometimes like...

From the very fact that he does not to have to create new documentary

could possibly be partially or completely absorbed by the "documentalist" sector of distribution, including commercially: sale of microfilms and search on demand are developing right now. 
"content" (of an empirical, extra-documentary origin) the documentalist is led to create "content" of an "interdocumentary" type. Because of the symbolism which he has created (e.g., classification), he may become a researcher of a superior type - one who combines. But this could only be through a lateral outcome of his current principal function as distributor, an outcome which would surely be accompanied by a division of labor.

\section{Current Situation of the Librarian}

That suffices to indicate the present state of the librarian's situation. Insofar as he now deals not only with books but also with periodicals - with all of written documentation - he appears as a special case of the documentalist. One must fear abuse of Hegelian metaphors, but it is the case to say that the documentalist goes beyond the librarian in integrating it, but also suppressing various characteristic aspects of his functions - notably the (unproductive) restrictive aspects...

It is rather artificial to distinguish the activity of the librarian from the activity of technical documentary production when it concerns books. In fact, historically, it was often the librarians who have themselves sprung out of their shadow, that is to say outside of the definition which limited them before the general development of documentation.

In the context of a comment already made, according to which "documentalist" designates a professional genus rather than a species, it would be logical to consider the librarian as a documentalist specialized, consciously or not, in a particular sector, in the same way as an archivist, museographer, ... or "film librarians." The general education as a documentalist could become a shared prerequisite for these specialties, at the same time as preparation for the specialized work of documentary coordination.

\section{Alterations to the "Book" Form}

630 In fact, following the above analyses, the current situation of the librarian includes a process of a different scope, which concerns 1) the symbolism of language, 2) the book as a documentary form, and 3) the library as a documentary organization.

\section{Instability of Classic Language (Book Content)}

I have indicated that language is drawn between two tendencies at its extremities: the introduction into languages of international techno-scientific vocabulary and syntax prepares them for profound changes. 


\section{Instability of the Book Form}

The material form of the book is threatened on several sides.

\section{The Development of Periodicals and the Collectivization of Intellectual Work}

Intellectual work, which has long remained individual and artisanal, is more and more clearly taking on a technically cooperative form, with separation of operations and the division of labor. As a result, most publications are collective, whether in name or not. Either they come from organizations or defined groups, or they indicate use of shared materials (bibliographies). The tendency, as much among authors as documentalists, is to suppress wordy redundancy between publications which summarize prior work (repetition is replaced by references). On the other hand, methodical collective work makes possible regular, periodic output, delivered for consumption that itself tends to be periodic and regular.

Finally, the increase in the output of intellectual work requires rapid publications to avoid being out-of-date or useless duplication. The need for rapid information for rapid theoretical and practical response contributes to the same effect. The direct integration of culture into collective practice introduces the factors of production, hence speed and rhythm. Technologically, culture tends to be presented as a segment of the process of social work. But periodicals, like all collective works, do not respond to the earlier norms of library work. Today, although nearly all scientific and technical creation appears in periodicals $(800,000$ important scientific articles a year before the war of 1939), libraries rarely catalogued these articles, and certainly not rapidly. It is the documentation centers that do it. Libraries largely store cultural archives (outdated documents of historical interest).

\section{Combinatorial Flexibility: Cards and Loose Leaves}

The technology of loose leaves and cards increasingly displace registers, notebooks, logs, and books because of their expandability, correctability... in short because of their combinatorial properties being very superior to those of a book (with bound leaves). The mental combinatorial operation is replaced by physical equipment (multiplication of combinations using signs). These devices form the apparatus which have prepared and are preparing the mechanization of combinatorial operations. So, despite its appearance, a loose-leaf volume is much more like a card file than a book: it is functionally a document that can be 
continually or periodically, individually or collectively be kept up-to-date updated, searched in, and rearranged.

Treatises, encyclopedias, manuals... that is, the forms most enduringly tied in appearance to the book form, can only tend toward this type of "file," with permanent collective authorship, endowed with combinatorial flexibility.

\section{The Book as a Physical Form Favoring Sequential Argument}

One could suppose that works with sequential argument, of the type of contemporary mathematics, and anywhere the meaning of a fragment depends on its position, are more likely than others to survive in the format of bound leaves. But one sees, in this case, that the book retains one of its physical properties only by changing most of its usual content. On the other hand, bound leaves are only one form among others of all documents with fixed structure.

\section{Microfilm}

Microfilm seems to result from several tendencies: a reaction against the volume and weight of books and the press, to lighten and compact the cultural equipment of groups and of individuals, to facilitate transmission; a reaction to the unsuitability of the book to supply excerpts (better selectivity) or a very small number of copies, at the moment and the extent of needs (a reaction against characteristics of typographical publications: massive, infrequent, unsuited to needs).

On the other hand, the microfilm makes possible, in addition to excerpting, and the insertion of additional argumentation, a likely technical refuge for the book.

Its shortcoming seems to be its synoptic capabilities, possessed by the book and card file (scanning leaves), a weakness that it shares with the contemporary cinema. Every document that imposes a rhythm of unfolding, a direction and a moment for "reading" is thereby an obstacle to combinatorial operations.

Already efforts have been made to overcome this weakness and to obtain with film the equivalent of loose-leaf: stop, reverse... (Etienne Mollier, for educational film).

\section{Relative Retreat of the Book}

Awaiting the development of these processes, the book perhaps possesses an advantage whenever the file format is not superior to it.

We are not at the point where the "library" would contain only prototypes of microfilms. Especially since the preceding arguments are purely technical, and 
it is not certain that what is technically superior dominate completely or rapidly, whatever one may wish. (Conditions of "profitability" at such or such a level are like brakes).

However, today the relative retreat of the book is certain. The era of its hegemony is over, so is the primarily written culture that it defined. It is probable that the hegemony of the classic written techniques will suffer a decline: typography can only give ground to photographic (or equivalent) processes when it comes to mass reproduction of images or many non-alphabetical signs.

\section{Libraries and Document Centers}

Finally, productive cultural consumption is all the more efficient when it is based on more complete documentary sources and therefore less exclusively on written documentary sources. From this point of view, the material regrouping of documents, no longer primarily according to their physical format but by their cultural role, is at once desirable and hardly avoidable. The condition of cultural work assumes the accessibility of the materials to be worked on. This is another reason for the library, whose internal change I have shown, to become materially only a sector of the document center and to lose its material independence after its hegemony. The large national libraries already have discographic and iconographic annexes... this is only an indication, perhaps, of the fact that tomorrow they will not be "annexes."

\section{The Social and Cultural Effect of Documentary Evolution}

70 Words remain, content changes, and it is necessary to suppress arguments over words. "Library" and "librarian" no longer have the same meaning as they did yesterday, despite the traditional crystallization of their characteristics. As for the documentalist, on the other hand, the definition of what concerns him is all the more unclear and more expansive (excessively, as I have indicated) as his field is itself in the process of transformation and expansion. This crisis of definition is only the symptom of a crisis of organization and division of cultural labor, an element of a transformation, some aspects of which have been suggested.

\section{Industrialization of Intellectual Work}

The first effect of the current industrialization of intellectual work (often inspired by industrial administrative techniques) is to alter the old artisanal intellectual occupations, as much of the researcher as of the librarian, and to transform both into narrowly specialized workers, not individual owners of their own means of 
production. They are tied to a social and physical machinery ever more massive and complex over which the majority of them have no control but which is nevertheless susceptible to being "planned" as never before. In the face of this machinery, there is no appeal to conscience or intelligence that "goes beyond" it, because it is precisely this machinery which is the principal and most efficacious factor in today's human intelligence. Rather than lamenting or dreaming of lost paradises and lost geniuses (like Leonardo da Vinci), it is more worthwhile to analyze the process in order to seek a suitable, effective response.

\section{Cultural Effect and Social Effect of the Cultural Technical Evolution}

There is no doubt that the higher level of contemporary culture (with a dominant combinatorial sector) has achieved both the scale and the precision of direct or aided sensory experience, with the solidness, the efficacy and the combinatorial power of symbolism, and the mass, the variety and the degree of exploitation of the documentary accumulation. From the point of view of the cultural effect in the abstract, the balance is clearly positive, and it is not "progress" that we should speak of, but the permanent acceleration of production. If the old forms of symbolism (language) and of production and distribution (the book) lose their hegemony, it is serious only for those who cannot adapt to the process (the "bookish").

As to the concrete social effect, one aspect has been seen: the semiempirical cultural level of the masses and cultural differentiation have, without doubt, increased. To determine the correlations of this effect will be another more serious theoretical and practical problem.

However, that is enough to indicate the kind of contributions and problems that cultural technology and, notably, documentology, could propose to bring forward to the study of contemporary man.

\footnotetext{
i "All theory is gray, my friend. But forever green is the tree of life" (Johann Wolfgang von Goethe, Faust, First Part). André Gide used "throw away the book" to encourage independence.

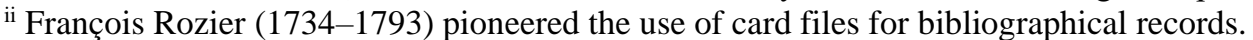

iii Thérèse Leroy, e.g. Méthodes de classement: La science et la classologie, ses applications. Le Prat, 1946.

iv La légende des siècles, a book of epic poems by Victor Hugo.

v French historian Numa Denis Fustel de Coulanges (1830-1889), noted for his emphasis on ancient texts, is associated with the dictum "no documents, no history."

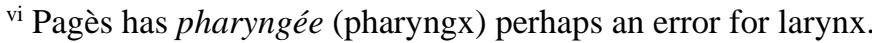

vii Gérard Cordonnier (1907-1977), French mathematician and inventor, worked on indexing and retrieval devices.

viii Philipp Frank (1884-1966) was a member and Moritz Schlick (1882-1936) the nominal leader of the Vienna Circle of Logical Positivists.
} 
${ }^{i x}$ Erlebnis is German for lived experience.

${ }^{\mathrm{x}}$ In French, experience and experiment are the same word: expérience.

${ }^{x i}$ Charles Perrault. Paralelle des anciens et des modernes: en ce qui regarde les arts et les sciences, 1688 , t. II, dial. III).

xii Édouard Claparède (1873-1940), Swiss neurologist, child psychologist, and educator.

xiii Pagès uses the word documentothèque. Here it evokes the library (bibliothèque).

${ }^{\text {xiv }}$ Fiches perforées is probably a reference to the use of optical coincidence ("peek-a-boo") cards as used, for example, by Gérard Cordonnier (1907-1977) and later by Pagès himself, but could also include edge-notched and tabulator "punch cards," which were in use by this time. 